\title{
Implementation of Low-Cost UHF RFID Reader Front-Ends with Carrier Leakage Suppression Circuit
}

\author{
Bin You, ${ }^{1}$ Bo Yang, ${ }^{1}$ Xuan Wen, ${ }^{2}$ and Liangyu $Q u^{3}$ \\ ${ }^{1}$ The Key Laboratory of RF Circuits and System of Ministry of Education, Hangzhou Dianzi University, Hangzhou 310018, China \\ ${ }^{2}$ Nokia Siemens Networks Technology (Beijing) Co., Ltd., Hangzhou 310053, China \\ ${ }^{3}$ Shanghai TYD Electronic Technology Co., Ltd., Shanghai 200233, China
}

Correspondence should be addressed to Bo Yang; jasonyoung327@gmail.com

Received 29 August 2013; Accepted 9 October 2013

Academic Editor: Bing Liu

Copyright (C) 2013 Bin You et al. This is an open access article distributed under the Creative Commons Attribution License, which permits unrestricted use, distribution, and reproduction in any medium, provided the original work is properly cited.

A new ultrahigh frequency radio frequency identification (UHF RFID) reader's front-end circuit which is based on zero-IF, single antenna structure and composed of discrete components has been designed. The proposed design brings a significant improvement of the reading performance by adopting a carrier leakage suppression (CLS) circuit instead of a circulator which is utilized by most of the conventional RF front-end circuit. Experimental results show that the proposed design improves both the sensitivity and detection range compared to the conventional designs.

\section{Introduction}

Although the theoretical basis of RFID technology has been established in 1948 [1], it took a long time before the technology advanced to current level. Unlike other wireless identification technology (such as bar codes and infrared technology), the UHF RFID uses backscattering communication which brings the noncontact, non-line-of-sight characteristics, and the long identified distance, high-speed reading, and other advantages make the UHF RFID becoming the most promising automatic identification technology around the world.

Nowadays, the development of UHF RFID front-end design has two trends: one is to use specific RFID ICs, such as Indy R2000 produced by Intel and AS3992 produced by austriamicrosystems; the other is to use discrete components. Using the specific ICs can shorten the development circle, while using the discrete components overcomes the limitation of protocols integrated in the specific ICs, makes the design more flexible and easier to optimize, and brings an enhancement of the performance and reduction of the cost. Thus, in today's market, most of the commercial UHF RFID reader's front-ends with high performances are designed by using discrete components.

In order to simplify the circuit structure, most of the RF front-ends are based on zero-IF and single-antenna structure.
Generally, the conventional RF front-end consists of four parts: the local oscillator (LO), transmitting circuit (Tx), receiving circuit $(\mathrm{Rx})$, and the circulator. Figure 1 shows the block diagram of a conventional RF front-end build by discrete components. As it is known that one distinctive characteristic of the UHF RFID reader is that it transmits the strong carrier wave and receives the faint backscattered signals of tags at the same frequency simultaneously [2]. Because of the imperfect $\mathrm{Tx}$ to $\mathrm{Rx}$ isolation of the circulator (usually $22 \mathrm{~dB}$ ), either a LNA or the receiver will be saturated by the strong Tx carrier wave leaks into the $\mathrm{Rx}$, and the LNA is abnegated before the receiver in the conventional frontend; thus, the sensitivity and the performance of the reader is degraded drastically.

In this paper, a redesigned RF front-end circuit of UHF RFID reader based on zero-IF and single-antenna structure and built by discrete components is proposed. The circuit of the proposed front-end is more simplified than the conventional one, due to the integration of the LO and the modulator by choosing a proper chip. Besides, the performance of the reader is also improved by using a newly designed carrier leakage suppression (CLS) circuit instead of the circulator, and adding a LNA before the receiver.

The structure of this paper is as follows. Section 2 gives the design of the proposed UHF RF front-end. Section 3 


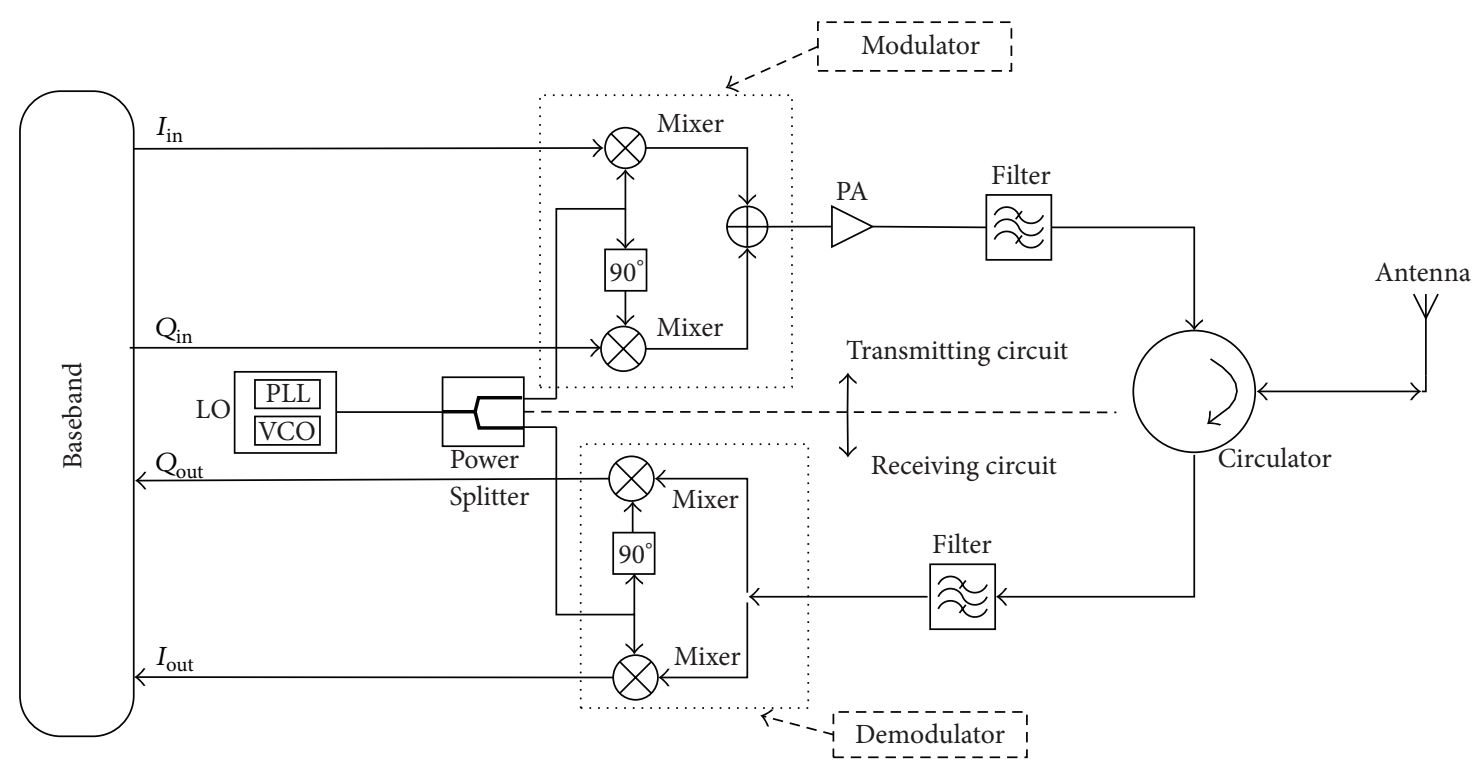

FIGURE 1: Block diagram of conventional RF front-end.

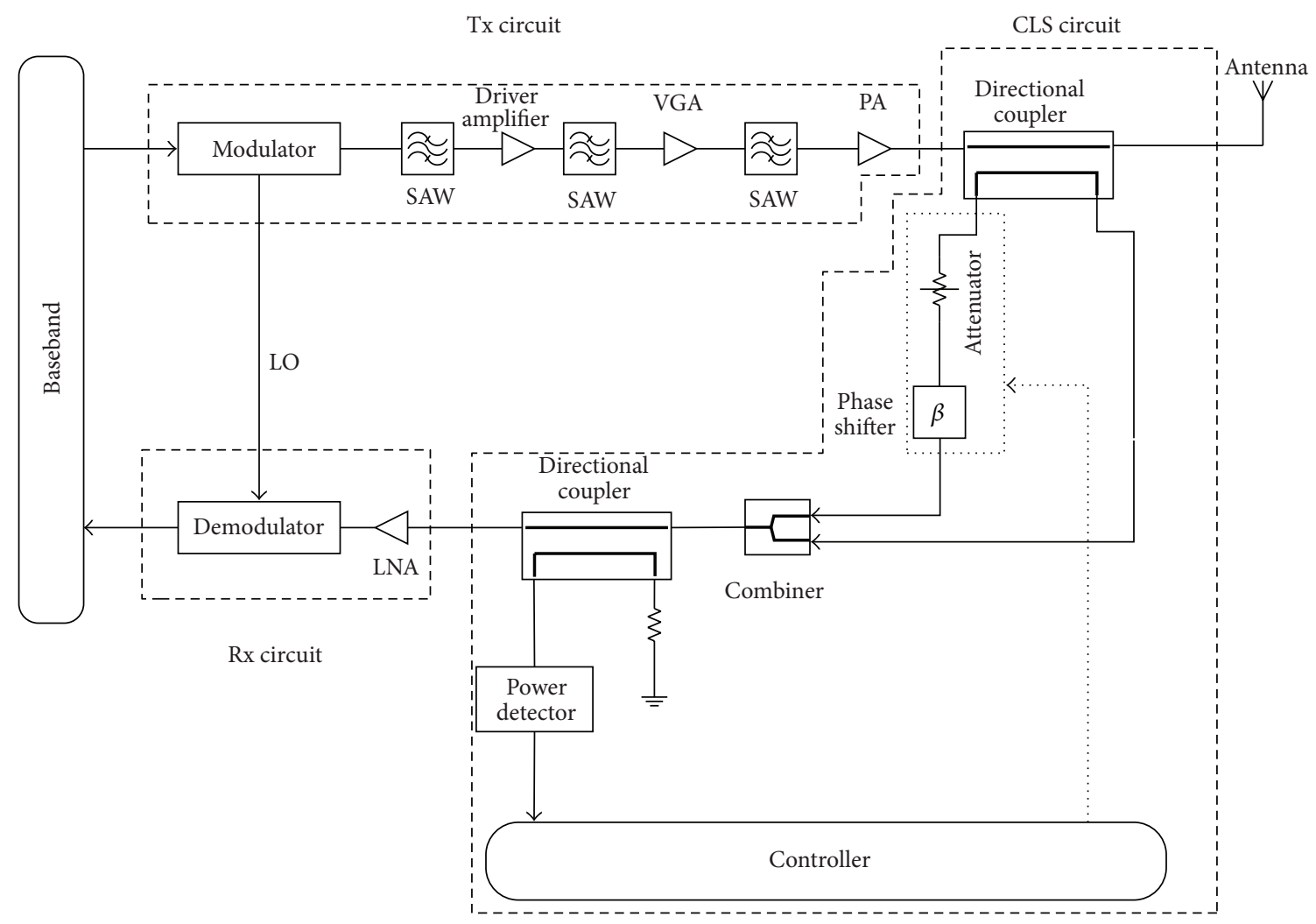

FIGURE 2: Block diagram of the proposed RF front-end.

demonstrates the implementation and the experimental results. The conclusion is drawn in Section 4.

\section{Design of the Proposed UHF RFID RF Front-End}

The proposed RF front-end is composed of three parts: Tx circuit, Rx circuit, and CLS circuit, as is shown in Figure 2.
2.1. Tx Circuit. The main function of the Tx circuit is to modulate baseband signal and amplify the modulated signal. In order to simplify the RF front-end circuit, the ADF9010 is chosen as the modulator. It is not only because of its high output power ( $-3 \mathrm{dBm}$ to $3 \mathrm{dBm}$ ) which can fulfill the input requirement of the later amplifier block, but also because of its integrated LO generation which is available at the output to drive an external RF demodulator. Moreover, the ADF9010 


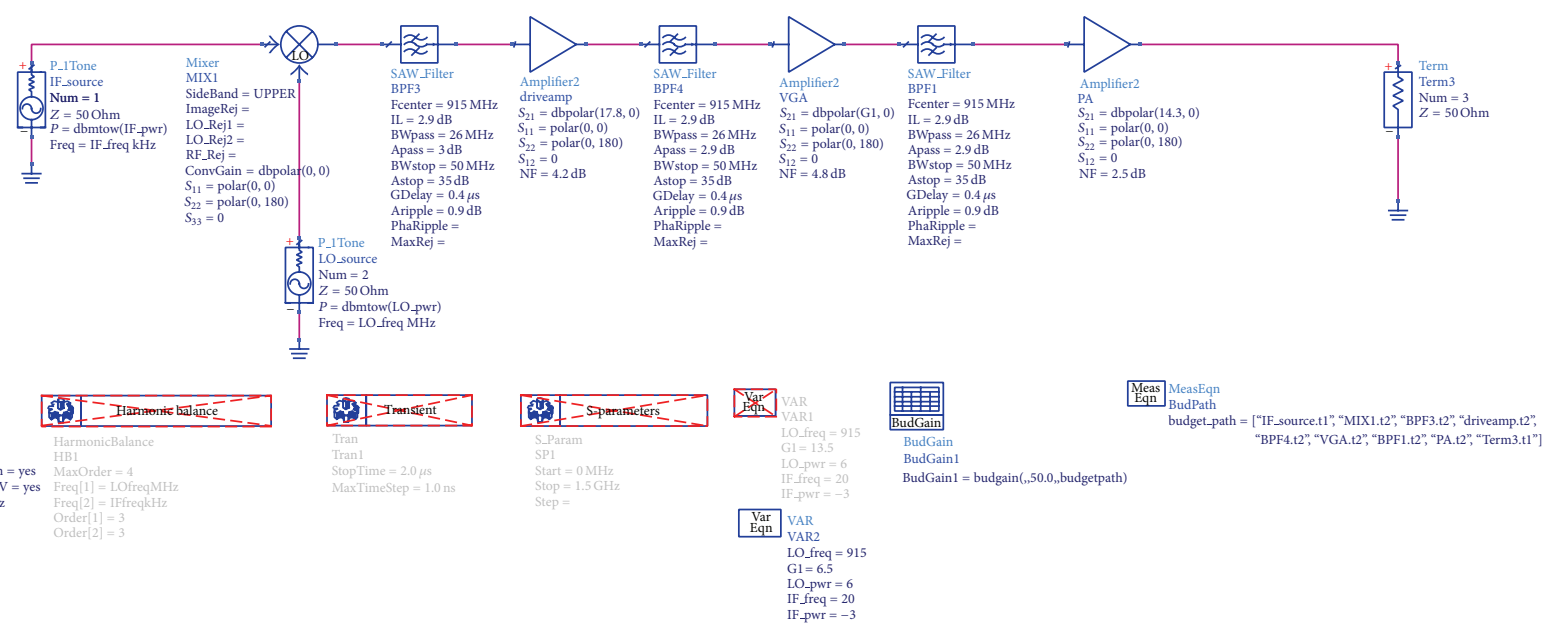

(a)

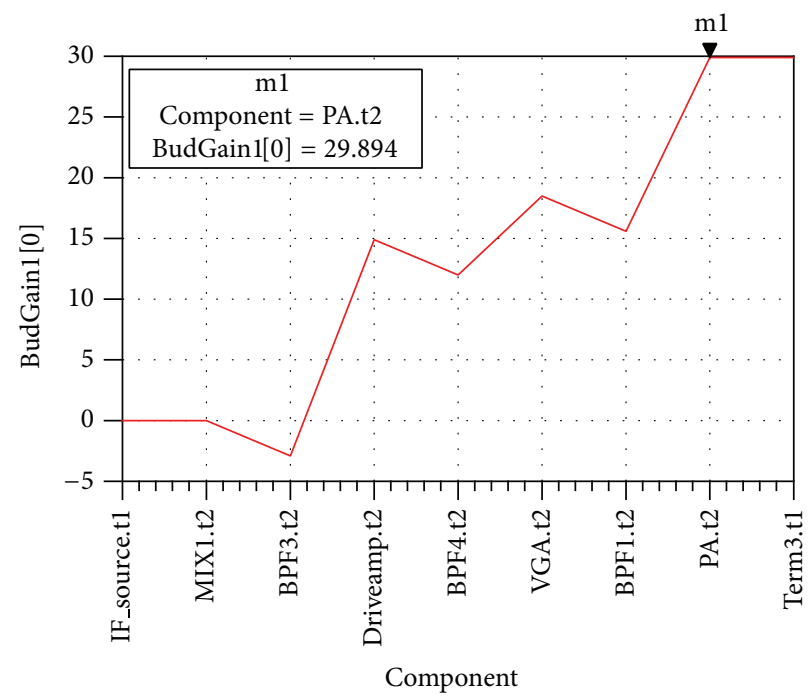

(b)

FIGURE 3: Link budget simulation of the proposed Tx circuit: (a) behavior model of the Tx circuit; (b) simulation result when VGA sets a $6.5 \mathrm{~dB}$ gain.

integrates a receive baseband PGA (Rx PGA) offering up to $24 \mathrm{~dB}$ voltage gain. The AVT54689 (Driver Amplifier), HMC80110 (VGA), and ALM-32120 (PA), along with the SAW filters, compose the amplifier block. Figure 3 gives the link budget simulation of the Tx circuit by using the Advanced Design System (ADS). Figure 3(a) is the behavior model of the Tx circuit, and Figure 3(b) is the simulation result. It shows that when the VGA with a gain control range of $-40 \mathrm{~dB}$ to $13.5 \mathrm{~dB}$ sets a $6.5 \mathrm{~dB}$ gain, the total gain of the Tx circuit can achieve nearly $30 \mathrm{~dB}$, which means if the ADF9010 sets a $0 \mathrm{dBm}$ output power, then the output power of the Tx can achieve $30 \mathrm{dBm}$ easily.

2.2. Rx Circuit. The ADL5382 is chosen as the demodulator. With $\mathrm{NF}=14 \mathrm{~dB}, \mathrm{IP} 1 \mathrm{~dB}=14.7 \mathrm{dBm}$, and IIP3 $=33.5 \mathrm{dBm}$ at $900 \mathrm{MHz}$, the ADL5382 offers an outstanding dynamic range which is suitable for the demanding infrastructure direct-conversion requirements. In order to lower the $\mathrm{Rx}$ circuit's noise figure, the ADL5523, a low noise amplifier (LNA) is added before the demodulator. Figure 4 gives the Link budget simulation of the Rx circuit. According to the read-range analysis of the UHF RFID reader provided by the material [3], the input power of the receiver is set to $-70 \mathrm{dBm}$ in the simulation, assuming that the distance between the passive tag and the reader's antenna is $10 \mathrm{~m}$. And the maximum gain of the Rx PGA in the ADF9010 is set.

The simulation result shows that the maximum gain of the $\mathrm{Rx}$ circuit can achieve nearly $74 \mathrm{~dB}$, which means when a passive tag is $10 \mathrm{~m}$ far from the reader's antenna, the output power of the baseband signal can be $4 \mathrm{dBm}$, which fulfills the input requirement of the analog to digital converter (ADC).

2.3. CLS Circuit. One distinctive characteristic of the UHF RFID reader is that it transmits the carrier wave signal 


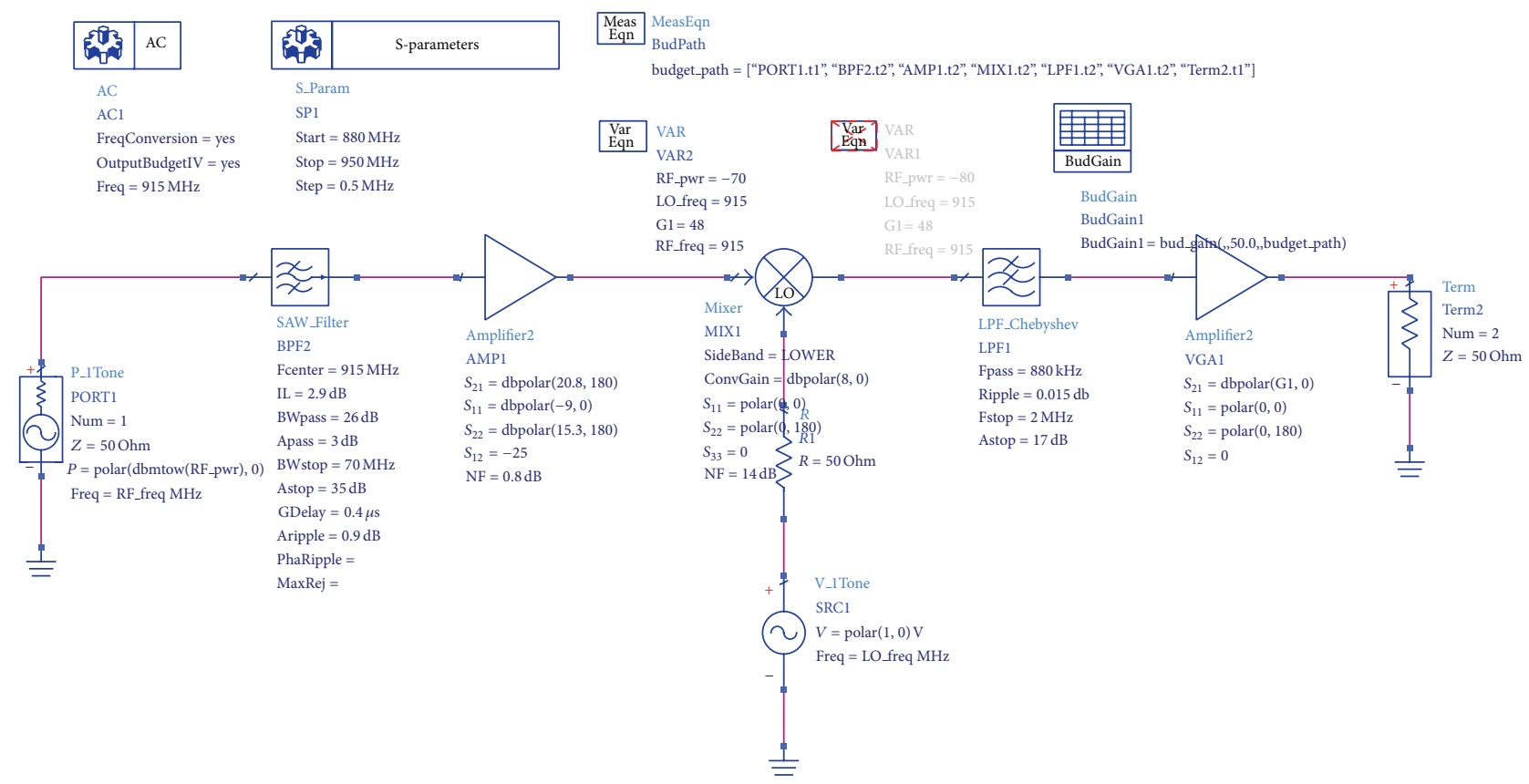

(a)

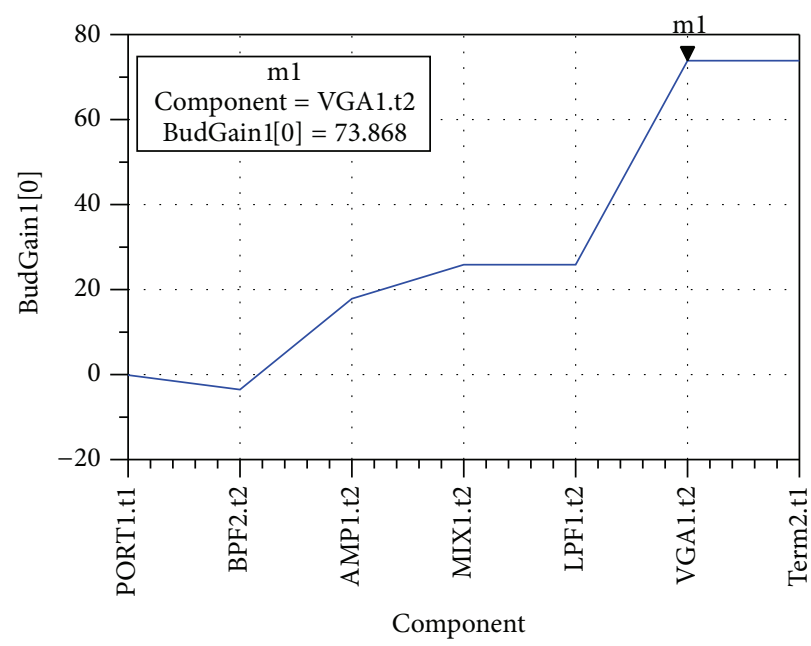

(b)

FIGURE 4: Link budget simulation of the proposed Rx circuit: (a) behavior model of the Rx circuit; (b) simulation result when the input power of the receiver is $-70 \mathrm{dBm}$, and the voltage gain of the Rx PGA of the ADF9010 is $24 \mathrm{~dB}$.

and receives the backscattered signal from tag in the same frequency band simultaneously. This characteristic results in that the reader's front-end circuit requires high isolation to prevent the transmitter's carrier signal from leaking into the receiver [4]. Thus in conventional RF front-end based on one antenna structure, a circulator or a directional coupler is used to isolate the $\mathrm{Tx}$ to $\mathrm{Rx}$; however the isolation characteristic in well matched condition of these devices can hardly exceed $25 \mathrm{~dB}$; moreover if the front-end is mismatched by an antenna, the isolation worsens [5]. In order to improve the isolation between $\mathrm{Tx}$ and $\mathrm{Rx}$, a newly designed CLS circuit is proposed. Figure 5 gives out the block diagram of the proposed CLS circuit.
As is shown in Figure 5, the carrier signal received by the receiver (LNA input) is consisted of signal A and signal B. The signal A is the carrier leakage signal, which is a vector sum of three signals: the first one is the carrier leaks to the isolation port (Port4) due to the imperfect isolation of the directional coupler; the second one is the reflected signal from the antenna because of the input impedance mismatch of the antenna; the third one is the reflected signal from the coupling port (Port3) of the directional coupler due to the imperfect matching between the attenuator and the coupling port. The single B is the coupled carrier signal. The principle of the proposed front-end is to properly adjust the magnitude and phase position of the coupled carrier signal 


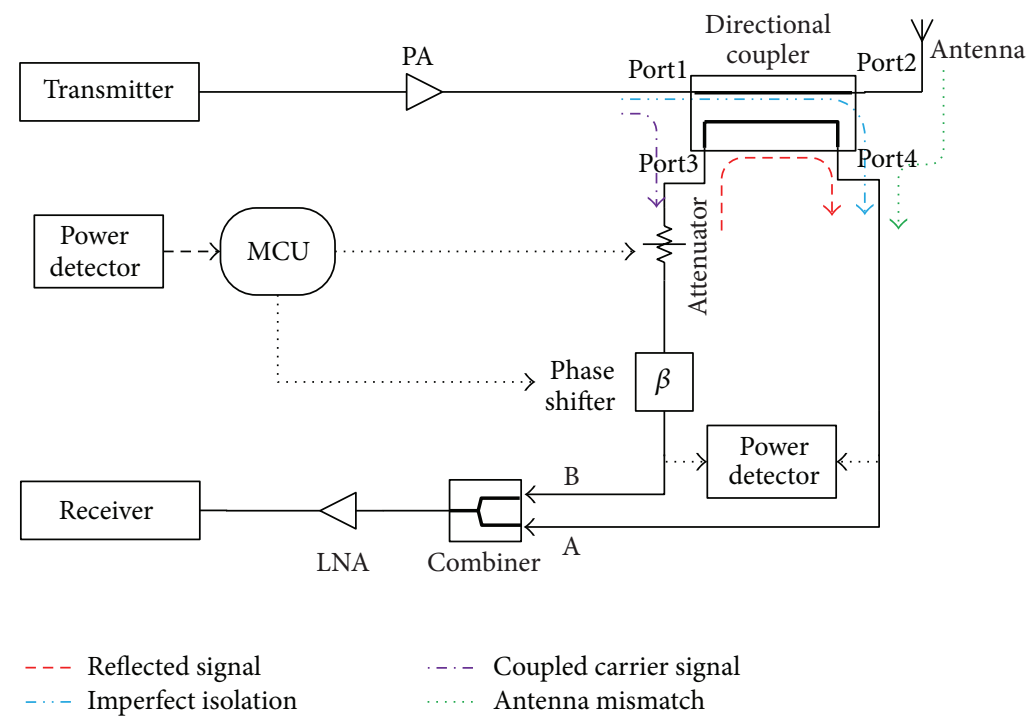

Figure 5: Block diagram of the proposed CLS circuit.

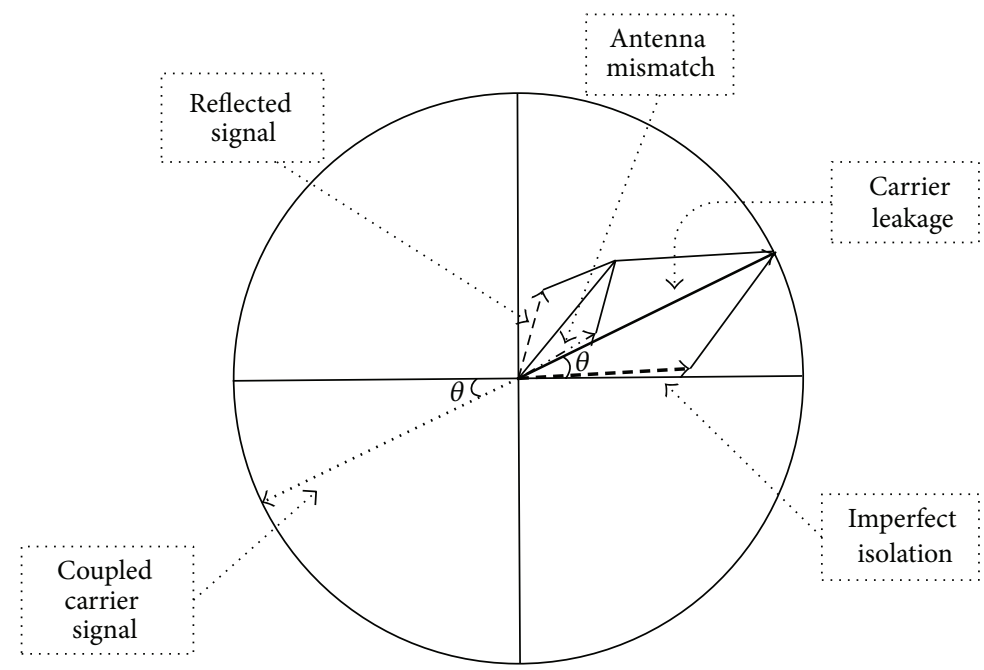

FIGURE 6: Vector representation of the carrier leakage signals and their cancellation using the coupled carrier signal.

by using the attenuator and the phase-shifter, to complete the carrier leakage suppression. Figure 6 shows the vector of the carrier leakage signals and their cancellation using the coupled carrier signal. Hence, as long as the operating frequency range of the attenuator and the phase-shifter can cover the UHF RFID frequency band, the proper cancellation can be achieve without worrying about the influence of the impedance matching of both the antenna and the attenuator.

\section{Implementation and Experimental Results}

In order to validate the performance of the proposed carrier leakage suppression (CLS) circuit, the CLS circuit has been fabricated. A 5 dB directional coupler of RN2's RCP890A05, an attenuator of Skyworks' AV101-12, which has a typical attenuation of $30 \mathrm{~dB}$, a phase-shifter of Skyworks' PS094-315 with a 80-110 degree phase shift range, and a combiner of M/A-COM's MAPDCC0001 are used. The antenna of Larid's A9028L30NF has a gain of $8 \mathrm{dBi}$ and an operating frequency range between 902 and $928 \mathrm{MHz}$, which is for one of the UHF RFID frequency bands. A photograph of the fabricated circuit is shown in Figure 7, including the schematic diagram of the experimental setup.

Figure 8 shows the isolation characteristics $\left(S_{21}\right)$, tested by using the Agilent-8719ES (S-Parameter Network Analyzer), of the fabricated CLS circuit and conventional circulator at the center frequency of $915 \mathrm{MHz}$, respectively. The test environment is set in the open area of the laboratory. Experimental result shows that the isolation between the transmitter $(\mathrm{Tx})$ and receiver $(\mathrm{Rx})$ is improved by more than $45 \mathrm{~dB}$ at the center and by at least $30 \mathrm{~dB}$ within $20 \mathrm{MHz}$ band from the center. Hence the leakage signal to the receiver can be removed almost totally. 


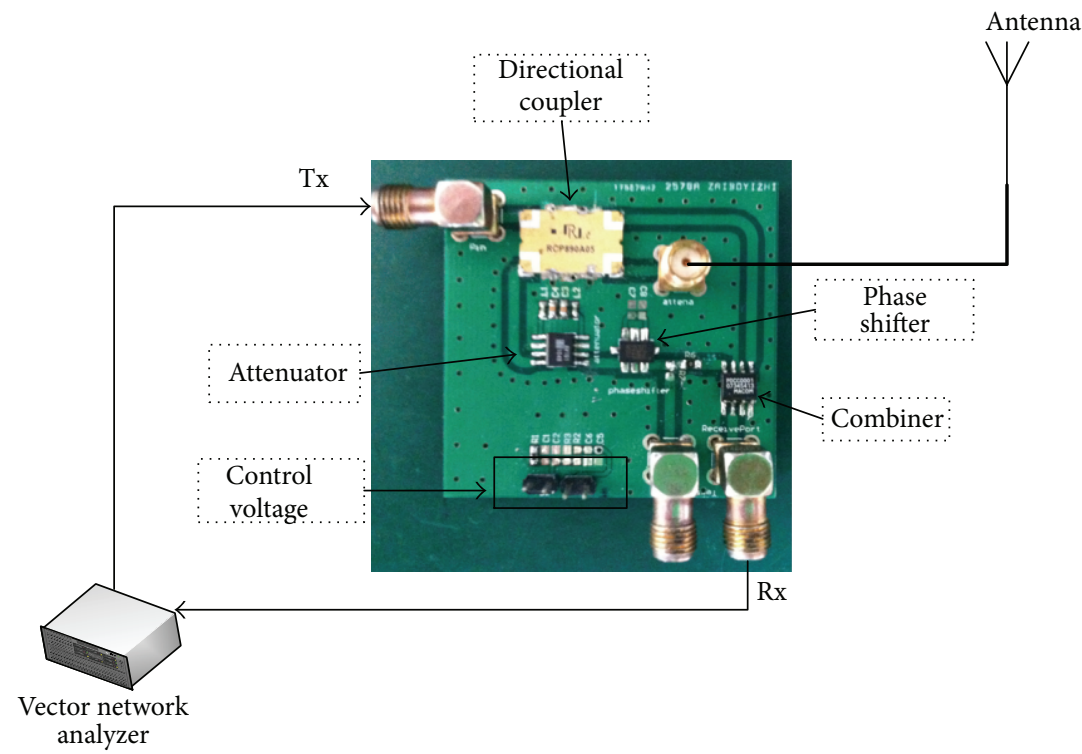

FIGURE 7: Photograph of the fabricated CLS circuit, including the schematic diagram of the experimental setup.

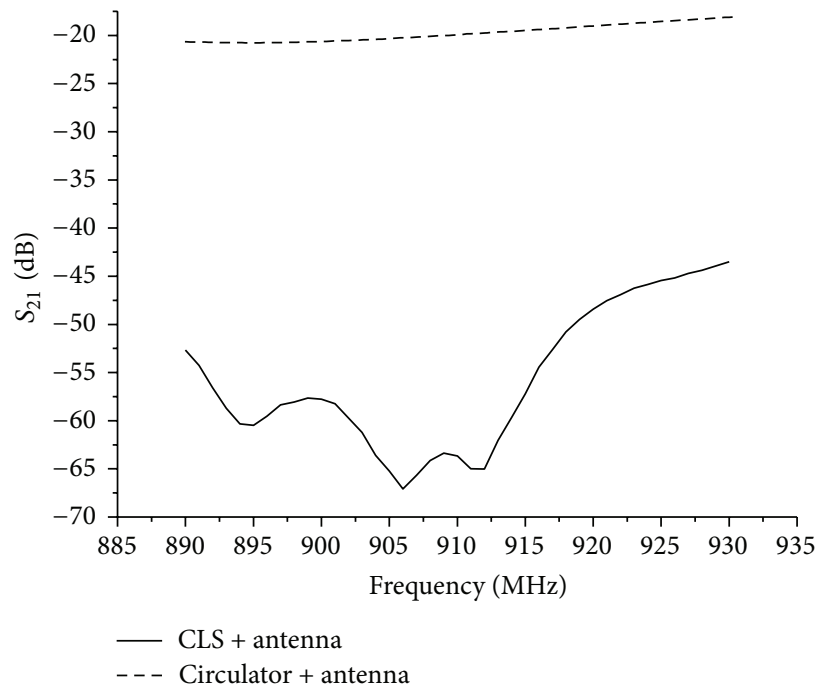

FIGURE 8: Isolation characteristics $\left(S_{21}\right)$ of the fabricated CLS circuit and circulator.

The fabricated board of the proposed UHF RFID reader's front-end is shown in Figure 9. The main components and their specifications are listed in Table 1.

Figure 10 gives out both the test result and the measurement setup of testing the received signal by using circulator and CLS circuit, respectively. The output power at the Tx Test Point (PA output) is $20 \mathrm{dBm}$, and the distance between the tag and the antenna is $10 \mathrm{~cm}$. In Figure 10(a) the CLS circuit in the fabricated frontend board shown in Figure 9 is disabled, and a circulator (MAFRIN0461) is added instead of the CLS circuit, the circulator's Portl is connected to the Tx Test Point (PA output), the Port2 is connected to the antenna, and the spectrum analyzer (Agilent-E4440A) connected to the Port 3 is used to measure the received signal. In Figure 10(b) the CLS circuit is enabled and the antenna is connected to the Antenna Port of the board, while the spectrum analyzer is connected to the Rx test pointl (LNA input). Figure 10(c) is the testing results of (a) and (b). As it is shown in Figure 10(c), if the circulator is used instead of the CLS circuit, there is a very high carrier leakage signal (approximately $7 \mathrm{dBm}$ ) leaking into the $\mathrm{Rx}$ test pointl (LNA input), which can result in degrading the reader's sensitivity or even saturating the LNA. However when the CLS circuit is used and the control voltages of both the attenuator and phase-shifter are tuned manually to achieve the maximum cancellation, the carrier leakage reduces, about $50 \mathrm{~dB}$. In addition, reading range measurement is setup in an open environment in the laboratory to see the reading distance by using the circulator and the CLS circuit, respectively. An Impinj's Monza 3 tag chip is used in the test. The tag, including 


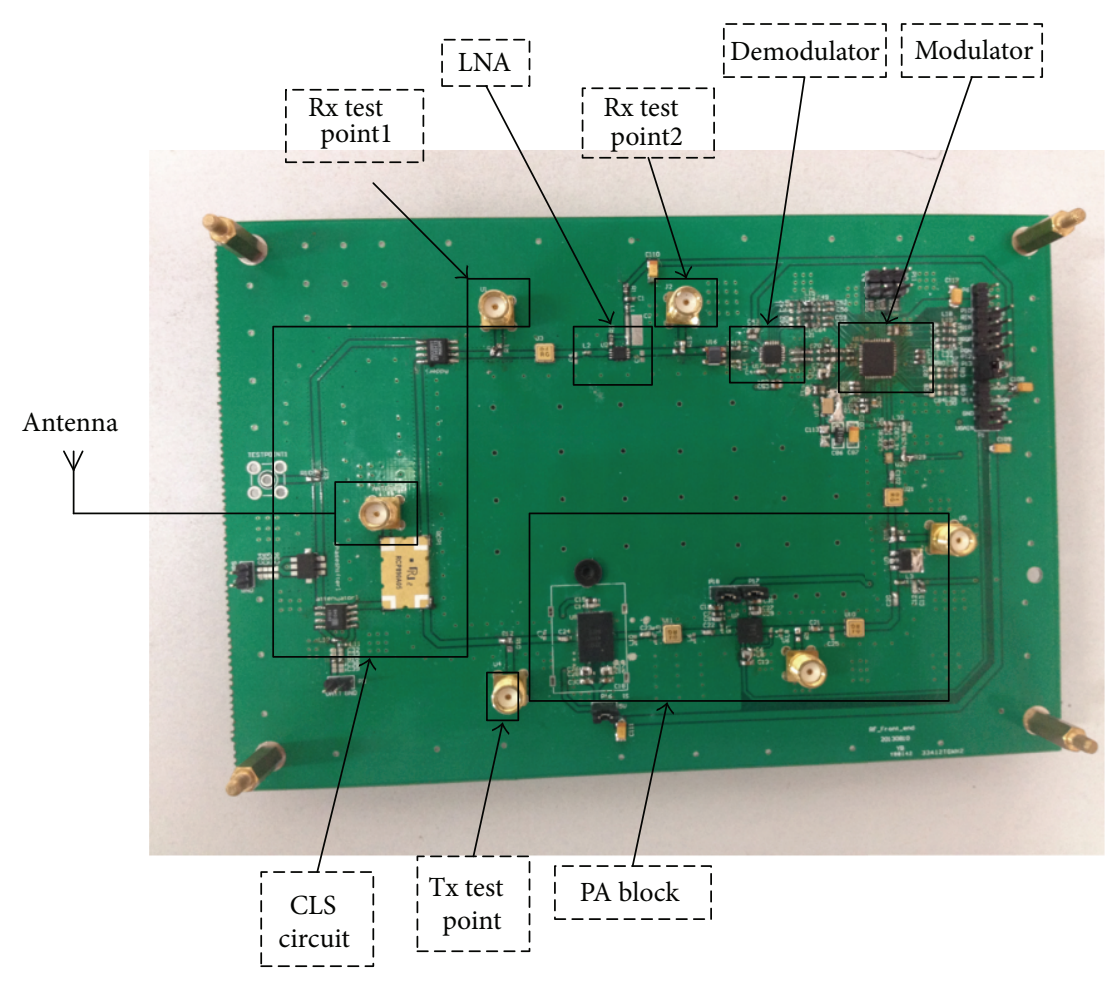

FIGURE 9: Photograph of the fabricated board.

TABLE 1: Summary of the components' specification.

\begin{tabular}{|c|c|c|}
\hline Part & Model & Specification \\
\hline Modulator & ADF9010 & $3 \mathrm{dBm}$ output power \\
\hline Demodulator & ADL5382 & 14.7 input $\mathrm{P} 1 \mathrm{~dB}$ \\
\hline Driver amplifier & AVT54689 & $\begin{array}{l}17.8 \mathrm{~dB} \text { gain and } 17.2 \mathrm{dBm} \\
\text { P1dB }\end{array}$ \\
\hline VGA & HMC80110 & $\begin{array}{l}13.5 \mathrm{~dB} \text { gain with a control } \\
\text { range of } 53.5 \mathrm{~dB} \text {, and } \\
23.2 \mathrm{dBm} \mathrm{P} 1 \mathrm{~dB}\end{array}$ \\
\hline PA & ALM32120 & $\begin{array}{l}14.3 \mathrm{~dB} \text { gain and } 34.4 \mathrm{dBm} \\
\mathrm{P} 1 \mathrm{~dB}\end{array}$ \\
\hline LNA & ADL5521 & $\begin{array}{l}20.8 \mathrm{~dB} \text { gain with a NF of } \\
0.8 \mathrm{~dB} \text {, and } 21.8 \mathrm{dBm} \mathrm{P} 1 \mathrm{~dB}\end{array}$ \\
\hline Directional coupler & RCP890A05 & $\begin{array}{l}5 \mathrm{~dB} \text { coupling and } 23 \mathrm{~dB} \\
\text { isolation }\end{array}$ \\
\hline Attenuator & AV101-12 & $30 \mathrm{~dB}$ attention \\
\hline Phase shifter & PS094-315 & $\begin{array}{l}80-110 \text {-degree phase shift } \\
\text { range }\end{array}$ \\
\hline Combiner & MAPDCC0001 & 0.5 -degree phase balance \\
\hline SAW & B3588U410 & $2.9 \mathrm{~dB}$ insertion loss \\
\hline
\end{tabular}

the antenna, is attached on a plastic board, and is moved away from the reader's antenna by $5 \mathrm{~cm}$ steps. Table 2 gives out the summary of the reading distance. As can be seen from the measure result, with the help of the CLS circuit, the distance of the reading range has been improved by about $27 \%$.
TABLE 2: Summary of the reading distance.

\begin{tabular}{lcc}
\hline \multirow{2}{*}{ Pout $(\mathrm{dBm})$} & \multicolumn{2}{c}{ Reading distance $(\mathrm{cm})$} \\
\hline 17 & Circulator & CLS \\
19 & 295 & 360 \\
21 & 325 & 420 \\
23 & 360 & 450 \\
\hline
\end{tabular}

\section{Conclusion}

An UHF RFID reader's front-end with a new designed CLS circuit has been proposed. The front-end is based on zeroIF scheme and composed of discrete components in order to simplify the structure of the circuit and reduce cost. The CLS circuit consisted of a directional coupler and a magnitude and phase tuning circuit is used to improve the isolation between the transmitter and the receiver of the proposed UHF RFID reader's front-end.

The isolation between the Tx and Rx in the proposed CLS circuit used in the proposed UHF RFID reader's front-end has been improved more than $45 \mathrm{~dB}$ at the frequency point of $915 \mathrm{MHz}$ and by at least $30 \mathrm{~dB}$ within $20 \mathrm{MHz}$ band from the center by using the CLS circuit. Although the experimental result of the isolation performance in the proposed frontend is not so excellent as that of the CLS circuit itself, the improvement of the reader's sensitivity can be clearly seen from the reading distance increasing. 


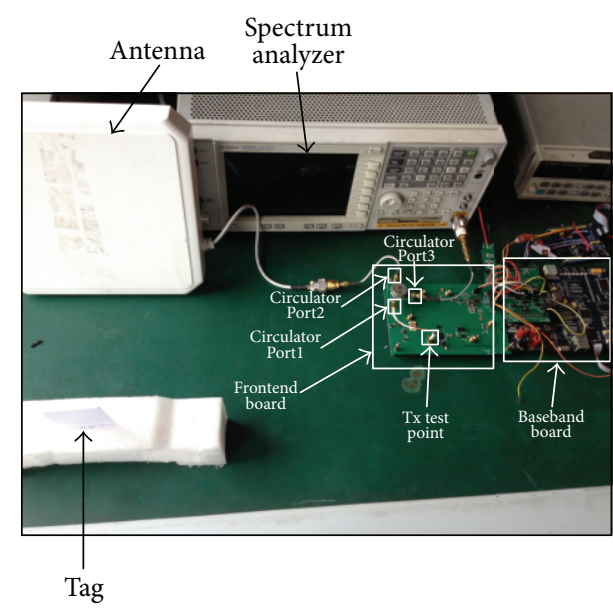

(a)

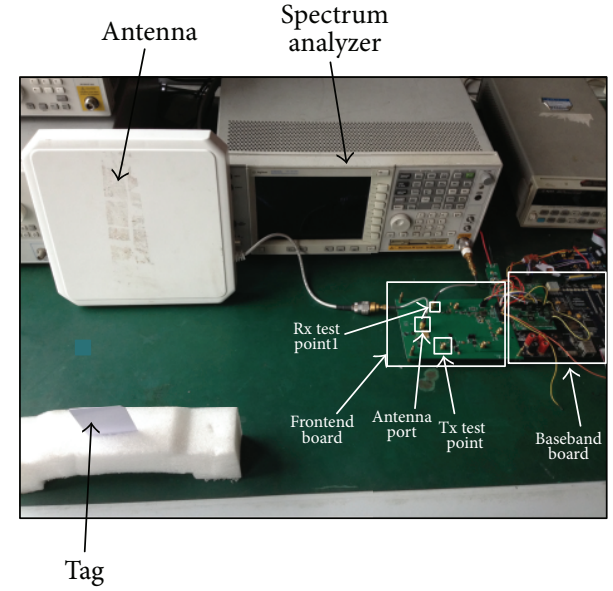

(b)

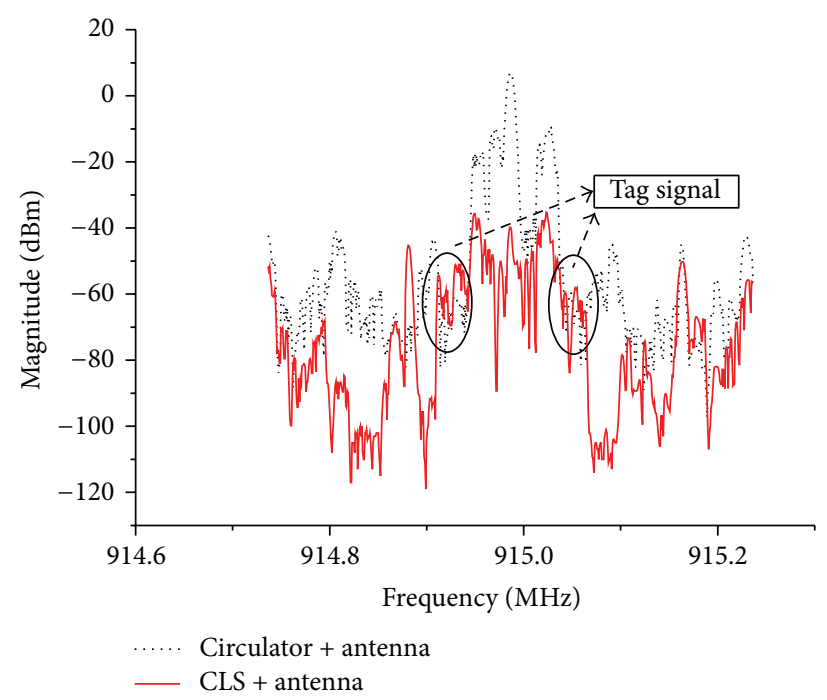

(c)

Figure 10: Measurement setup and testing result of the received signal of circulator and CLS circuit, respectively, at a Tx output power of $20 \mathrm{dBm}$; (a) measurement setup when testing the received signal by using circulator instead of CLS circuit; (b) measurement setup when testing the received signal by using CLS circuit; (c) testing result of (a) and (b).

\section{Conflict of Interests}

The authors declare that there is no conflict of interests regarding the publication of this paper.

\section{Acknowledgment}

This work was supported in part by Zhejiang Province Key Science and Technology Innovation Team (no. 2010R50010) and the Real-Time Anti-Theft System based on RFID technology (no. ZX130702307001).

\section{References}

[1] H. Stockman, "Communication by means of reflected power," in Proceedings of the IRE, pp. 1196-1204, October 1948.

[2] P. Bai, Y. Yin, and X. Yang, "A novel RX-TX front-ends for passive RFID reader with high isolation," in Proceedings of the
IEEE International Symposium on Microwave, Antenna, Propagation, and EMC Technologies for Wireless Communications (MAPE '07), pp. 332-335, August 2007.

[3] J.-Y. Jung, C.-W. Park, and K.-W. Yeom, "A novel carrier leakage suppression front-end for UHF RFID reader," IEEE Transactions on Microwave Theory and Techniques, vol. 60, no. 5, pp. 1468-1477, 2012.

[4] A. Safarian, A. Shameli, A. Rofougaran, M. Rofougaran, and F. De Flaviis, "RF identification (RFID) reader front ends with active blocker rejection," IEEE Transactions on Microwave Theory and Techniques, vol. 57, no. 5, pp. 1320-1329, 2009.

[5] W.-G. Lim, S.-Y. Park, W.-I. Son, M.-Q. Lee, and J.-W. Yu, "RFID reader front-end having robust Tx leakage canceller for load variation," IEEE Transactions on Microwave Theory and Techniques, vol. 57, no. 5, pp. 1348-1355, 2009. 

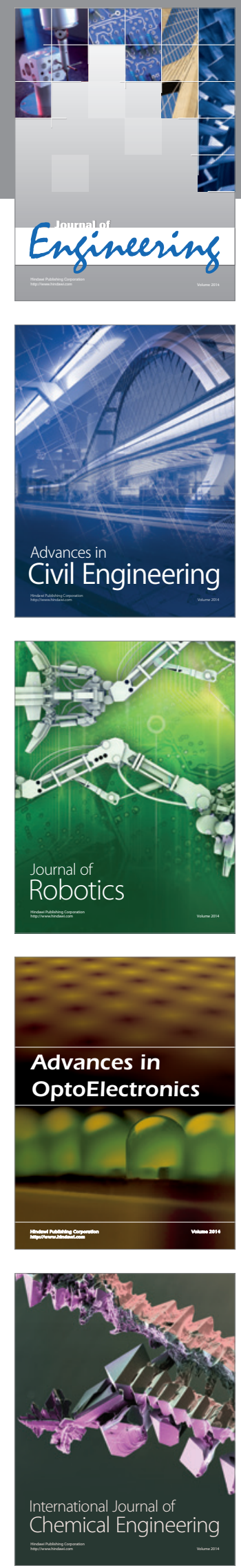

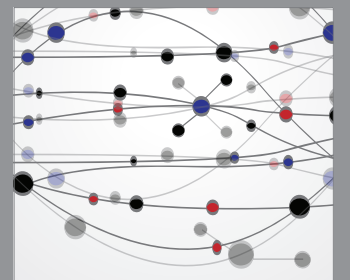

The Scientific World Journal
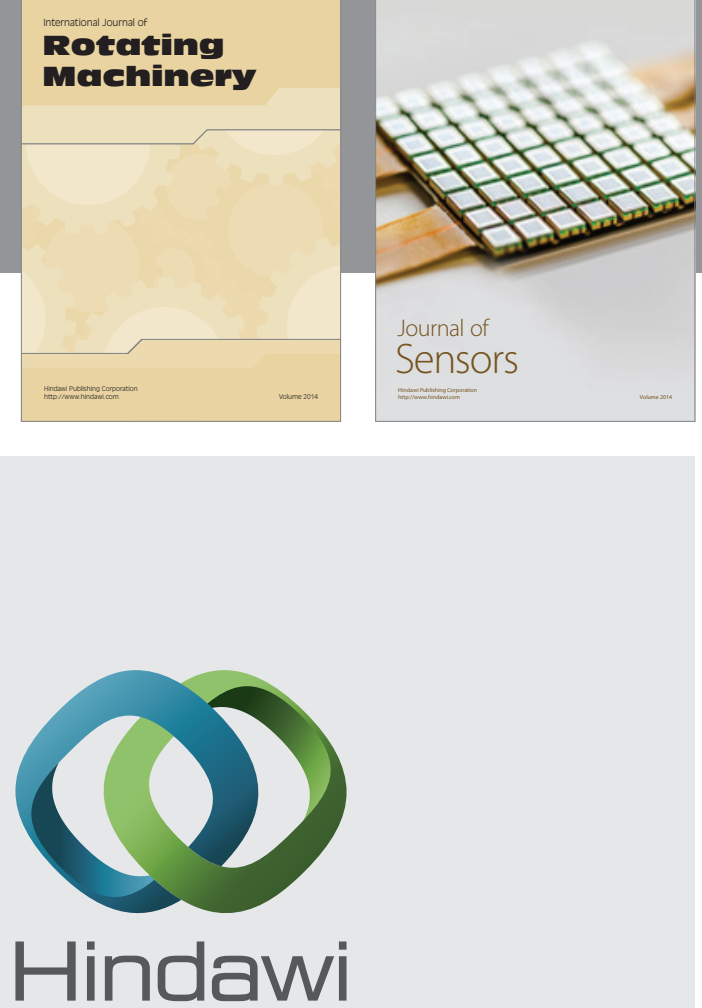

Submit your manuscripts at http://www.hindawi.com
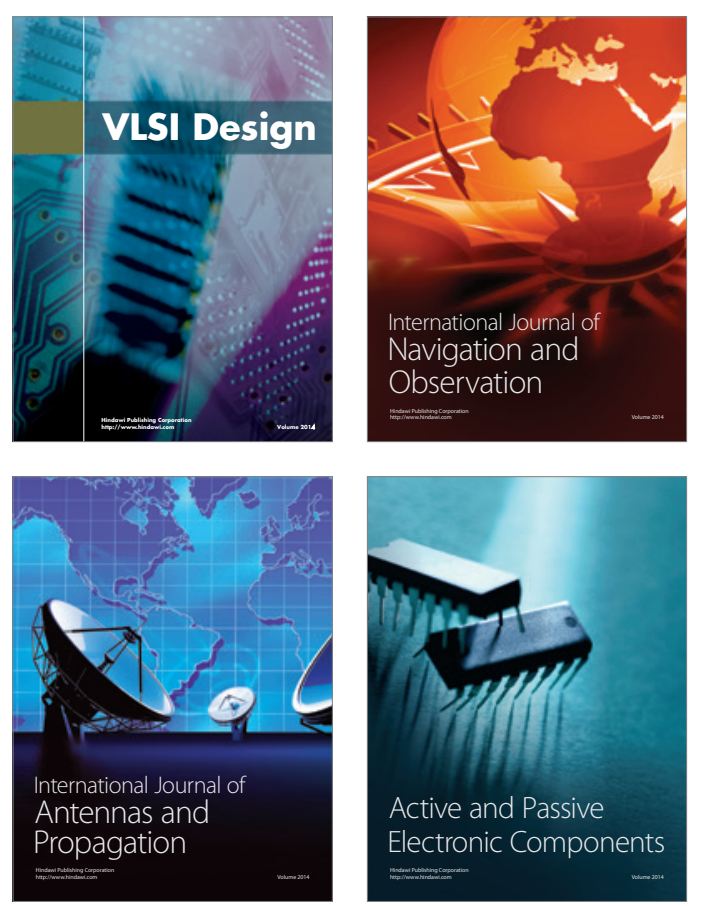
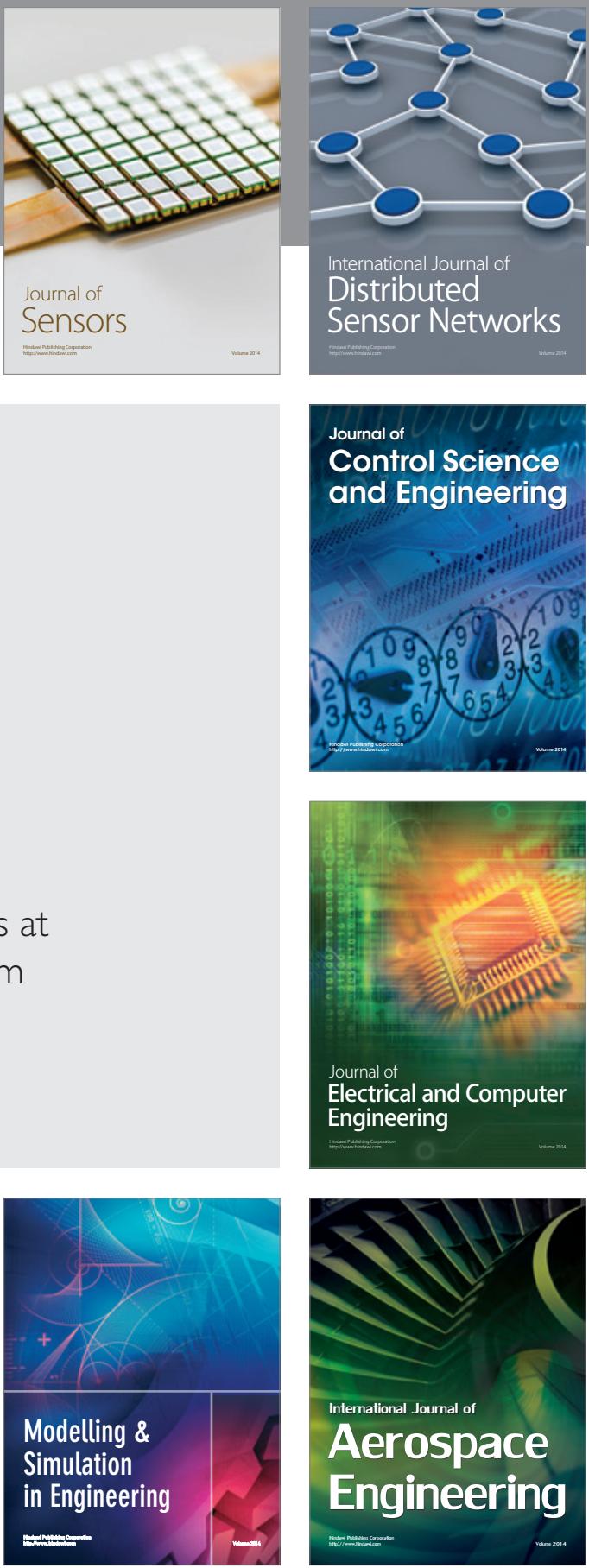

Journal of

Control Science

and Engineering
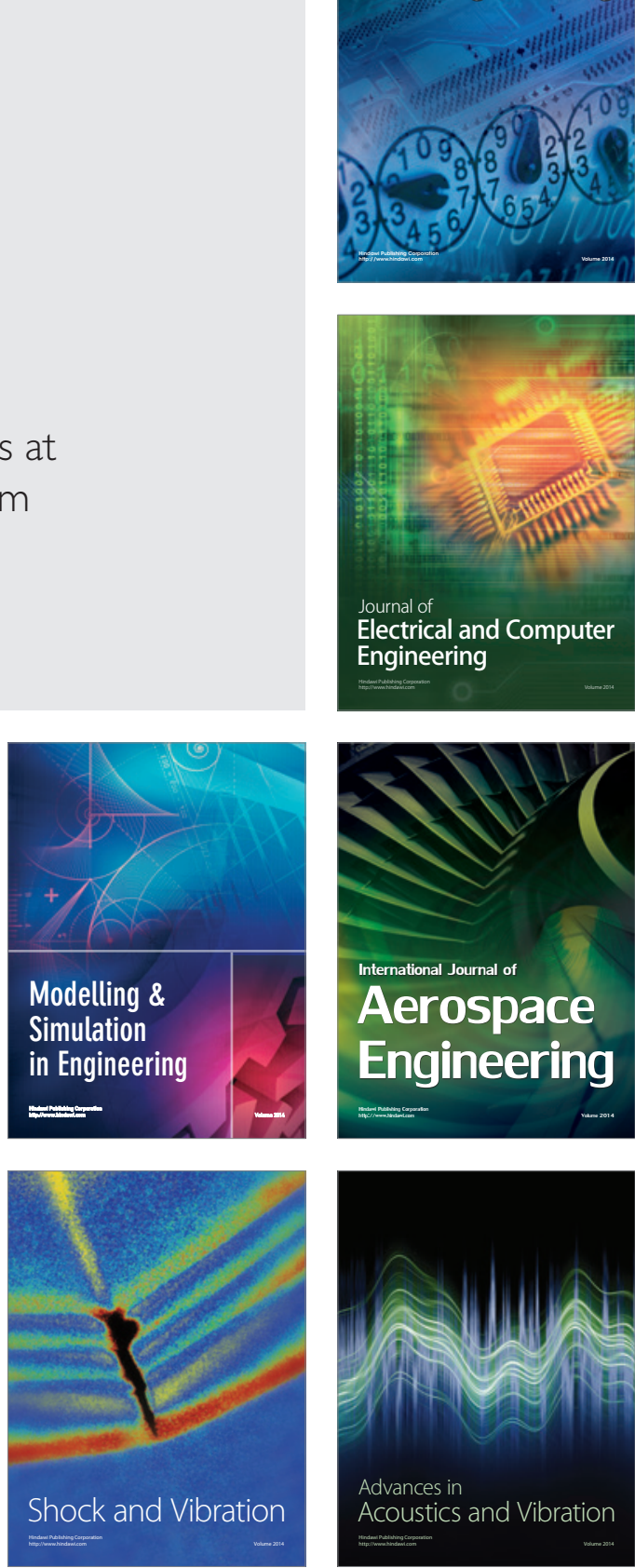